\title{
COMPARAÇÃO ENTRE OS EFEITOS DO USO DE DIFERENTES ADOÇANTES ARTIFICIAIS COMO SUBSTITUTO PARCIAL DO AÇÚCAR SOBRE O PROCESSO DE GELATINIZAÇÃO DO AMIDO EM BOLOS TIPO ESPONJA
}

\author{
A. V. de O. BARBOSA ${ }^{1}$ e C. E. M. da SILVA ${ }^{1}$ \\ 1 Universidade Federal do Ceará, Departamento de Tecnologia de Alimentos \\ E-mail para contato: albavaleriaob@yahoo.com.br
}

RESUMO - O efeito do uso de diferentes adoçantes artificiais, como substituto do açúcar, em bolos, vem sendo estudado, pois a redução do açúcar influi negativamente nos processos de formação estrutural dos bolos. Por atuar como agente selante, o atraso na gelatinização do amido, proporcionado pelo açúcar fortalece a estrutura da massa, controlando a formação da estrutura porosa característica. Objetivou-se comparar os efeitos do uso de sucralose e acessulfame K em níveis crescentes de substituição ao açúcar sobre a gelatinização do amido em bolos esponja, avaliando qual adequou-se, mais como substituto. $\mathrm{O}$ resultados obtidos demostraram que o uso dos respectivos adoçantes como substituto do açúcar afetaram negativamente a gelatinização do amido elevando as temperaturas (To e Tp) isso retardou acima do esperado a gelatinização do amido na massa, ocorrendo a redução demasiada da viscosidade da massa permitindo o escape das células de ar, restringindo a expansão da massa, os baixos valores de Tf, resultaram no decréscimo da $\Delta \mathrm{T}$, sugerindo que houve um estreitamento da faixa de gelatinização, associado a altos valores de $\Delta \mathrm{H}$ são um indicativo do enrijecimento da estrutura da massa, impedindo a expansão do ar ocasionando a redução do volume desejado. Esse efeito negativo provocado pela substituição do açúcar foi mais pronunciado nas formulações contendo acessulfame $\mathrm{K}$, concluindo que entre os dois adoçantes estudados esse é o menos indicado para tal finalidade.

\section{INTRODUÇÃO}

Bolos tipo esponja são produtos da panificação apreciados em todo mundo e por conter altas proporções de açúcar, são candidatos ideais de pesquisas que almejam o desenvolvimento de produtos com reduzido teor calórico para o consumo de pessoas com doenças crônicas ligadas ao consumo excessivo de açúcar como a obesidade e o diabetes Kocer et al., (2007).

No entanto a redução substancial do açúcar nos bolos afeta suas propriedades estruturais e sensoriais. Pois o açúcar exerce papel fundamental na formação estrutural dos bolos, pois rege todos os fenômenos relacionados como, o retardamento da gelatinização do amido, devido a sua capacidade de ligar-se a agua, formando pontes entre as cadeias de amido, e na desnaturação proteica (Baeva et al., 2003; Cavalcante, 2012). As proteínas desnaturadas são responsáveis pela armação estrutural da massa, com o atraso da gelatinização do amido este atua como agente selante, mantendo as células de ar bem distribuídas e fixadas a estrutura, fortalecendo-a, é o responsável pela formação porosa característica dos bolos tipo esponja (Kocer et al., 2007; Schimer et al., (2012). 


\section{9 a 22 de outubro de 2014 \\ Florianópolis/SC}

Bennion e Bamford (1997) afirmaram que a substituição é possível desde que substitua o açúcar por um adoçante de alta intensidade e um espessante de baixa caloria, pois o uso isolado de um adoçante em formulações de bolos diet não substitui por si só as funções do açúcar conferindo apenas doçura ao produto. Portanto, o uso combinado de um agente espessante (hidrocoloide) e um adoçante são imprescindíveis para corrigir os problemas ocasionados pela ausência do açúcar Baeva et al., (2000). O uso de diferentes adoçantes artificiais na formulação de bolos como substitutos do açúcar são exaustivamente como alternativa para a redução das calorias dos bolos, mantendo a sua doçura característica Cavalcante (2012).

A sucralose é um adoçante sintetizado a partir da sacarose em que três grupos hidroxilas, nas posições 4, 1 e 6 foram substituídos por 3 átomos de cloro resultando no composto 1,6-dicloro-1,6 dideoxi- $\beta$-D-fructofuranosil-4-Cloro-4-deoxi- $\alpha$-D-galactopiranose, com poder adoçante 600 vezes superior a sacarose (Barndt e Uackson, 1990) por ser estável a altas temperaturas e a acidez elevada, mantendo sua doçura durante o cozimento, seu uso como substituto do açúcar é recomendado em produtos de panificação (Lin e Lee, 2005; Martinez-Cevera et al., 2012). O acesulfame-K (C4H4N04KS) ou sal de potássio é um adoçante sintético não calórico e não metabolizado pelo organismo, com capacidade adoçante 180-200 vezes superior à da sacarose, possui a capacidade de resistir ao armazenamento prolongado e a diferentes temperaturas, tornando-o adequado para aplicações em alimentos, que incluem em seu processamento, etapas de aquecimento, como em produtos de panificação como bolos, tortas e biscoitos (Attia et al., 1993).

Objetivou-se com esse estudo, comparar os efeitos do uso de sucralose e acessulfame K em níveis crescentes de substituição ao açúcar sobre a gelatinização do amido em bolos esponja, avaliando qual adequou-se, mais como substituto.

\section{MATERIAL E MÉTODOS}

\section{1-Material}

Para o preparo do bolo controle, usado para fins comparativos utilizou-se ingredientes convencionais adquiridos, no comércio de Fortaleza: Farinha de trigo Tipo 1 sem fermento, açúcar cristal, margarina sem sal, ovos tamanho médio de cor branca, leite em pó integral, fermento químico em pó e água mineral.

No preparo dos bolos com reduzido teor calórico, além dos ingredientes convencionais utilizou-se também os adoçantes: Sucralose, cedido gentilmente pela TATE \& LYLE (São Paulo-SP) e o acessulfame K, cedido generosamente pela M. Cassab (São Paulo-SP) utilizados como agentes de doçura, selecionados pela sua resistência a altas temperaturas, e a goma xantana usada como agente de volume foi fornecida pela Gastronomy lab (Brasília-DF).

\section{3-Métodos}

Preparo dos bolos Para o preparo dos bolos com teor calórico reduzido, utilizou-se uma formulação controle otimizada por Cavalcante (2012) apresentada na tabela 01, Foram elaboradas 7 
(sete), mantendo fixas as proporções dos ingredientes, farinha de trigo, leite em pó, fermento químico, margarina, variando apenas a quantidade de açúcar no qual foi substituído em reduções crescentes pela solução dos adoçantes a $1 \%$ e goma xantana a 1,5\%, totalizando 7 formulações para cada adoçante, com os mesmos níveis de substituição, para facilitar a comparação entre ambos, cuja codificação baseou-se no nível de substituição do açúcar pela solução dos adoçantes e goma xantana de acordo com o exposto na tabela 02 , sendo F0, a formulação controle contendo apenas o açúcar na formulação.

Tabela 01- Formulação de bolo tipo esponja otimizada

$\begin{array}{cc}\text { Ingredientes } & \text { Gramas } \\ \text { Farinha } & 100 \\ \text { Açúcar } & 155,88 \\ \text { Margarina } & 28,78 \\ \text { Leite em pó } & 6,79 \\ \text { Ovos } & 46,96 \\ \text { Fermento químico } & 1,00 \\ \text { Água } & 59,59\end{array}$

Tabela 02- Formulações de bolos preparadas com substituição do açúcar pela solução de adoçante (sucralose / acessulfame K) e goma xantana

\begin{tabular}{|c|c|c|c|}
\hline $\begin{array}{l}\text { Codificação } \\
\text { das } \\
\text { formulações }\end{array}$ & $\begin{array}{l}\text { Nível de substituição } \\
\text { do açúcar pela solução } \\
\text { adoçante e goma (\%) }\end{array}$ & $\begin{array}{l}\text { Proporção de } \\
\text { açúcar } \\
(\mathrm{g})\end{array}$ & $\begin{array}{c}\text { Proporção de solução } \\
\text { de adoçante e goma } \\
\text { (g) }\end{array}$ \\
\hline Controle (F0) & 0,00 & 155,88 & 0,00 \\
\hline F10 & 10,00 & 140,28 & 31,17 \\
\hline F19 & 19,00 & 126,27 & 59,23 \\
\hline $\mathrm{F} 27$ & 27,10 & 113,64 & 84,49 \\
\hline F34 & 34,39 & 102,28 & 107,21 \\
\hline $\mathrm{F} 40$ & 40,95 & 92,05 & 127,66 \\
\hline F46 & 46,86 & 82,83 & 146,09 \\
\hline F52 & 52,17 & 74,56 & 162,64 \\
\hline
\end{tabular}

Definida os formulações, procedeu-se o preparo dos bolos, onde utilizou-se o método rápido de preparo no qual consiste em adicionar e bater os ingredientes em uma só etapa sem acarretar prejuízos a formação estrutural do bolo, depois de misturados os ingredientes foram batidos em batedeira comum com 3 velocidades por 1 (um) minuto na velocidade 1, para permitir a incorporação de ar necessária para o desenvolvimento dos bolos, depois de batidos os ingredientes, a massa obtida foi vertida em forma tipo $\mathrm{W}$ e levada para assar em forno elétrico previamente aquecido por 30 minutos a $180^{\circ} \mathrm{C}$, a massa foi assada a mesma temperatura por aproximadamente 40 minutos e resfriadas a temperatura ambiente e armazenados em recipientes plásticos sob refrigeração. Antes de 


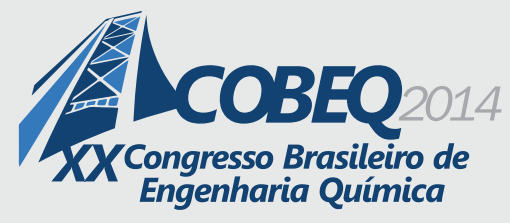

19 a 22 de outubro de 2014
Florianópolis/SC

serem levadas para o assamento, amostras das massas foram retiradas inclusive da formulação controle, realizando-se posteriormente a analise térmica (gelatinização do amido), depois de assados efetuou-se a medição do volume especifico da fatia dos bolos.

Análise das propriedades térmicas (gelatinização do amido) da massa do bolo tipo esponja Segundo metodologia adotada por Wilderjans et al. (2008). Para a obtenção das propriedades térmicas da massa, as amostras foram analisadas em um calorímetro diferencial exploratório (DSC) modelo 200F3 da Netzsch, com amostras de até $8,0 \mathrm{mg}$ das massas dos bolos com redução de açúcar, em cadinhos de alumínio selados, tendo como referência um cadinho de alumínio selado vazio, com uma velocidade de aquecimento de $10^{\circ} \mathrm{C} / \mathrm{min}$ no intervalo de temperatura de $20-250^{\circ} \mathrm{C}$. As características da gelatinização do amido nas massas de bolo em um termograma de DSC foi indicada por diferentes temperaturas: To (temperatura inicial de gelatinização) e Tf (Temperatura final de gelatinização), Tp (Temperatura de pico, Temperatura de fluxo máximo de calor diferencial), $\Delta \mathrm{T}$ (obtida pela diferença entre a temperatura final e a temperatura inicial) representa a faixa de temperatura de gelatinização e entalpia $\Delta \mathrm{H}$ (quantidade de energia necessária para completar o processo de gelatinização). Análise feita com três repetições.

Volume especifico dos bolos Depois de assado os bolos, efetuou-se o quarteamento para melhor representatividade da amostra, efetuando em seguida a medição do volume especifico da fatia dos bolos segundo metodologia descrita pela AACC (2000), por deslocamento da semente de painço. Análise feita em triplicata.

Tratamento estatístico Os resultados obtidos com as análises térmicas da massa dos bolos e o volume especifico dos bolos, foram submetidas à análise estatística, para tanto, usou-se o Delineamento Inteiramente Casualizado (DIC), onde foram feitas análises de variância (ANOVA) e cálculo do desvio das médias com posterior comparação das diferenças entre as médias pelo teste de Tukey a um nível de significância de p<0,05, utilizando o programa estatístico Statistica versão 8.

\section{0-RESULTADO E DISCUSSÃO}

Os resultados expostos na tabela 03 demostram que as massas de bolo contendo a sucralose e goma xantana como substituto do açúcar diferiram significativamente entre si, para valores de To $\left(60,6-88,5^{\circ} \mathrm{C}\right)$ e $\mathrm{Tp}\left(105,6-110,1^{\circ} \mathrm{C}\right)$, superiores a controle com exceção das formulações $\mathrm{F} 10$ e F19, no entanto valores de $\mathrm{Tf}\left(127,2-124^{\circ} \mathrm{C}\right)$, foram inferiores, salvo a $\mathrm{F} 34$ com valor igual a controle a controle excetuando F10 e F19. No que concerne às massas de bolo contendo acessulfame K e goma xantana, todos os valores de To $\left(74,2-85,6^{\circ} \mathrm{C}\right)$ e $\mathrm{Tp}\left(105,6-110,1^{\circ} \mathrm{C}\right)$, foram superiores ao controle, observando o mesmo fato relatado nas massas de bolo com substituição do açúcar por s sucralose, os valores de $\operatorname{Tf}\left(126,7-123,9^{\circ} \mathrm{C}\right)$ foram inferiores, excetuando a $\mathrm{F} 10 \mathrm{com}$ valor igual a controle.

Segundo Gaonkar e Mcpherson (2005) todos os adoçantes têm a capacidade de influenciar o ponto de gelatinização do amido, elevando a temperatura de gelatinização, esse fato é atribuído à competição dos adoçantes pela agua presente, já que os mesmos possuem afinidade maior que o amido e consequentemente, há menos água disponível para hidratar e gelatinizar o amido, alterando o ponto de gelatinização. 


\section{9 a 22 de outubro de 2014 \\ Florianópolis/SC}

Tabela 03:Medida das Temperaturas inicial (To), Temperatura de pico (Tp) e Temperatura final (Tf) de gelatinização do amido em massas de bolo com substituição parcial do açúcar por sucralose e goma xantana e das massas de bolo com substituição parcial do açúcar por acessulfame e goma xantana

\begin{tabular}{lcccllll} 
& \multicolumn{2}{c}{ To $\left({ }^{\circ} \mathrm{C}\right)$} & \multicolumn{2}{c}{ Tp $\left({ }^{\circ} \mathrm{C}\right)$} & \multicolumn{2}{c}{ Tf $\left({ }^{\circ} \mathrm{C}\right)$} \\
F0 & Sucralose & Acessulfame & Sucralose & Acessulfame K Sucralose & Acessulfame K \\
F10 & $60,6^{\mathrm{b}} \pm 0,21$ & $63,6^{\mathrm{g}} \pm 0,21$ & $101,7^{\mathrm{e}} \pm 0,14$ & $101,7 \pm 0,14$ & $126,8^{\mathrm{c}} \pm 0,08$ & $126,8^{\mathrm{a}} \pm 0,08$ \\
F19 & $57,3^{\mathrm{b}} \pm 0,11$ & $74,2^{\mathrm{f}} \pm 0,16$ & $90,8^{\mathrm{c}} \pm 0,12$ & $94,3^{\mathrm{f}} \pm 0,18$ & $105,6^{\mathrm{a}} \pm 0,18$ & $127,2^{\mathrm{b}} \pm 0,07$ & $126,7^{\mathrm{a}} \pm 0,20$ \\
F27 & $76,2^{\mathrm{b}} \pm 0,12$ & $79,8^{\mathrm{d}} \pm 0,07$ & $109,8^{\mathrm{b}} \pm 0,18$ & $107,8^{\mathrm{a}} \pm 0,15$ & $128,9^{\mathrm{a}} \pm 0,14$ & $121,5^{\mathrm{d}} \pm 0,30$ \\
F34 & $74,3^{\mathrm{b}} \pm 0,11$ & $78,7^{\mathrm{e}} \pm 0,14$ & $102,9^{\mathrm{d}} \pm 0,11$ & $107,4^{\mathrm{a}} \pm 0,07$ & $126,4^{\mathrm{c}} \pm 0,14$ & $123,4^{\mathrm{b}} \pm 0,15$ \\
F40 & $82,2^{\mathrm{b}} \pm 0,11$ & $85,3^{\mathrm{a}} \pm 0,15$ & $107,8^{\mathrm{c}} \pm 0,25$ & $108,5^{\mathrm{a}} \pm 0,22$ & $121,7^{\mathrm{d}} \pm 0,18$ & $121,8^{\mathrm{d}} \pm 0,15$ \\
F46 & $73,4^{\mathrm{b}} \pm 0,22$ & $83,3^{\mathrm{b}} \pm 0,14$ & $98,7^{\mathrm{g}} \pm 0,12$ & $108^{\mathrm{a}} \pm 0,18$ & $123^{\mathrm{e}} \pm 0,07$ & $122,4^{\mathrm{c}} \pm 0,20$ \\
F52 & $88,5^{\mathrm{a}} \pm 0,14$ & $85,6^{\mathrm{a}} \pm 0,14$ & $110,5^{\mathrm{a}} \pm 0,18$ & $110,1^{\mathrm{b}} \pm 0,18$ & $124^{\mathrm{d}} \pm 0,07$ & $123,6^{\mathrm{d}} \pm 0,20$ \\
\end{tabular}

Os resultados são apresentados como valores médios \pm desvio padrão. Valores seguidos por letras sobrescritas diferentes na mesma coluna são significativamente diferentes $(P<0,05)$ onde $a>b>c>d$ $>\mathrm{e}>\mathrm{f}>\mathrm{g}>\mathrm{h}$.

Essa alteração no ponto de gelatinização, como mostra na tabela 04 refletiu nos valores de $\Delta \mathrm{H}$, nas massas de bolo com substituição do açúcar por sucralose foram superiores ao controle variando de 138-747,8J/g com exceção das formulações F10, F19 e F34, que obtiveram valores inferiores ao controle. As massas de bolo com substituição do açúcar por acessulfame $\mathrm{K}$ obtiveram valores de $\Delta \mathrm{H}$ que variaram de $430,3-698,3^{\circ} \mathrm{C}$ superiores a controle. A diferença de temperatura $(\Delta \mathrm{T})$ que representa a faixa de temperatura de gelatinização do amido também foi afetada, Com base nesses valores, observa-se um decréscimo conforme elevou-se o nível de substituição do açúcar, em ambas as amostras de massa contendo os respectivos adoçantes e goma xantana (sucralose, $71,3-35,3^{\circ} \mathrm{C}$ ) e (acessulfame $\mathrm{K}, 49,3-38,4^{\circ} \mathrm{C}$ ) a valores inferiores a controle, exceto para as formulações F10 e F19 das massas com substituição do açúcar por sucralose e goma xantana indicando que houve um estreitamento na faixa de gelatinização do amido.

Essa capacidade de competir pela água do sistema, permitindo a alteração do ponto de gelatinização, foi observado em ambas formulações dos adoçantes selecionados para estudo no entanto, verificou-se que as massas de bolo com substituição parcial do açúcar por acessulfame K apresentou temperaturas superiores, inclusive nas formulações com níveis menores de substituição o que não foi observado nas massas contendo sucralose como substituto do açúcar, influindo na $\Delta \mathrm{H}$ de gelatinização que é a quantidade de energia gasta para completar esse processo, sugerindo que o acessulfame $\mathrm{K}$ apresentou capacidade superior a sucralose de competir pela água do sistema, portanto uma maior quantidade de energia foi requerida, elevando assim as temperaturas de gelatinização, com exceção da Tf, ocorrendo o estreitamento da faixa de gelatinização que por sua vez foi mais acentuado nas formulações contendo acessulfame $\mathrm{K}$ com valores reduzidos já nas formulações com menor nível de substituição. 


\section{9 a 22 de outubro de 2014 \\ Florianópolis/SC}

A elevação na entalpia de gelatinização do amido foi associado por Psimouli e Oreopoulou (2012) ao enrijecimento da estrutura da massa, suprimindo o desenvolvimento das células de ar, aumentando a pressão de vapor interna ocasionando a coalescência das células limitando a expansão da massa dos bolos.

Verificou-se com base nos dados apresentados na tabela 05 , que houve redução no volume especifico, os bolos contendo sucralose e goma xantana variaram de $1,90-1,06 \mathrm{~cm}^{3} / \mathrm{g}$, em relação ao controle $\left(1,81 \mathrm{~cm}^{3} / \mathrm{g}\right)$, com exceção da F10, tendo um decréscimo a partir da formulação F27, nos bolos contendo acessulfame $\mathrm{K}$ e goma xantana o volume especifico foi inferior ao controle $(1,81$ $\mathrm{cm}^{3} / \mathrm{g}$ ), onde os valores variaram de 1,90-1,06 $\mathrm{cm}^{3} / \mathrm{g}$, exceto a formulação F10, tendo um decréscimo a partir da F19. Isso é um indicio de que os valores superiores de entalpia apresentados pelas formulações contendo acessulfame $\mathrm{K}$ influíram na expansão da massa reduzindo o volume final dos bolos.

Tabela 04: Valores de $\Delta \mathrm{T}$ (Tf-To), $\Delta \mathrm{H}$ (entalpia) de gelatinização do amido das massas de bolo com substituição parcial do açúcar por sucralose e goma xantana e das massas de bolo com substituição parcial do açúcar por acessulfame e goma xantana.

$\Delta \mathrm{T}\left({ }^{\circ} \mathrm{C}\right)$

Sucralose AcessulfameK

F0

F10

$71,3^{\mathrm{a}} \pm 0,20$

F27 $50,3^{\mathrm{d}} \pm 0,1$

F34 $50,4^{\mathrm{d}} \pm 0,30$

F40 $38,7^{\mathrm{f}} \pm 0,2$

F46 $49,4^{ \pm} \pm 0,20$

F52 $35,3^{\mathrm{g}_{ \pm}} 0,2$ $63,2^{\mathrm{a}} \pm 0,20$

$49,3^{\mathrm{b}} \pm 0,20$

$40,4^{\mathrm{d}} \pm 0,25$

$43,5^{\mathrm{c}} \pm 0,30$

$43,3^{\mathrm{c}} \pm 0,2$

$37,3^{\mathrm{f}} \pm 0,2$

$38,3^{\mathrm{e}} \pm 0,2$

$38,4^{\mathrm{e}} \pm 0,2$
$\Delta \mathrm{H}(\mathrm{J} / \mathrm{g})$ Sucralose Acessulfame K $180^{\mathrm{e}} \pm 0,70$

$138^{\mathrm{g}} \pm 1,00$

$129^{\mathrm{h}} \pm 1,08$

$177^{\mathrm{f}} \pm 0,70$

$298,7^{\mathrm{d}} \pm 0,11$

$505,1^{\mathrm{b}} \pm 0,07$

$410,9^{\mathrm{c}} \pm 0,12$

$747,8^{\mathrm{a}} \pm 0,15$ $180^{\mathrm{h}}+0,70$

$430,3^{\mp} \pm 0,20$

$578,5^{\mathrm{c}} \pm 0,30$

$495^{\mathrm{e}} \pm 0,15$

$572,5^{\mathrm{d}} \pm 0,10$

$185,7^{\mathrm{g}} \pm 0,15$

$630,3^{\mathrm{b}} \pm 0,15$

$698,3^{\mathrm{a}} \pm 0,30$
Volume Especifico $\left(\mathrm{cm}^{3} / \mathrm{g}\right)$

Sucralose Acessulfame K

$1,81^{\mathrm{b}} \pm 0,01 \quad 1,81^{\mathrm{b}} \pm 0,01$

$1,90^{\mathrm{a}} \pm 0,09$

$1,80^{\mathrm{b}} \pm 0,03$

$1,90^{\mathrm{a}} \pm 0,02$

$1,59^{\mathrm{c}} \pm 0,06$

$1,51^{\mathrm{c}} \pm 0,05$

$1,19^{\mathrm{d}} \pm 0,03$

$1,50^{\mathrm{c}} \pm 0,01$

$1,17^{\mathrm{d}} \pm 0,01$

$1,29^{\mathrm{d}} \pm 0,02$

$1,16^{\mathrm{d}} \pm 0,02$

$1,05^{\mathrm{e}} \pm 0,03$

$1,19^{\text {de }} \pm 0,02$

$1,10^{\mathrm{f}} \pm 0,03$

$1,06^{\mathrm{f}} \pm 0,03$

Os resultados são apresentados como valores médios \pm desvio padrão. Valores seguidos por letras sobrescritas diferentes na mesma coluna são significativamente diferentes $(\mathrm{P}<0,05)$ onde $a>b>c>d>e>f>g>h$.

No entanto, observa-se que a partir da formulação F27, os valores de volume especifico são semelhantes nos bolos com substituição do açúcar por sucralose e goma xantana e os bolos com substituição do açúcar por acessulfame K e goma xantana, sugerindo que o enrijecimento da estrutura da massa em consequência os altos valores de entalpia não é a causa isolada da redução demasiada do volume especifico dos bolos. Ronda et al. (2005) afirmaram que a redução do açúcar em formulações de bolos causa prejuízos prontamente detectáveis na sua estrutura, e uma das causas apontadas foi a perca de estabilidade da massa durante a fase de aquecimento devido a redução da viscosidade. Durante o aquecimento a viscosidade da massa do bolo, diminui o que é desejável, pois permite que as células de ar provenientes da mistura dos ingredientes e da fermentação química na qual libera $\mathrm{CO}_{2}$, se expandam, quando inicia-se a gelatinização do amido controlada pela presença do açúcar, ocorre o aumento da viscosidade da massa, que impede o escape das células, retendo-as e aprisionando-as, formando assim a estrutura porosa com o volume desejado. 


\section{9 a 22 de outubro de 2014 \\ Florianópolis/SC}

Em estudo realizado por Lin e Lee (2005) a substituição gradual do açúcar por dextrina e sucralose provocou a elevação da temperatura de gelatinização, resultando na diminuição da viscosidade da massa durante o assamento, reduzindo a capacidade de retenção de ar, produzindo bolos com um menor volume indesejável. O aumento demasiado da temperatura, retardou acima do esperado a gelatinização do amido permitindo assim que as células de ar escapem, devido a redução da viscosidade, resultando em bolos de volume reduzido (Psimouli e Oreopoulou, 2012; Ronda et al., 2011). Portanto os dois adoçantes usados como substituto do açúcar, possuem a capacidade de alterar negativamente o processo de gelatinização do amido nas massas de bolo e consequentemente a formação estrutural dos bolos, mesmo apresentando valores de volume especifico semelhantes aos bolos contendo sucralose, verificou-se que o decréscimo do volume especifico dos bolos contendo acessulfame K logo na formulação F19, enquanto que nos bolos contendo sucralose, foi observado somente a partir da formulação F27.

Isso pode ser justificado pelo fato do acessulfame K ser um sal que segundo Miller e Wistler (2009) os sais apresentam a capacidade de elevar a temperatura de gelatinização do amido. A sucralose por se tratar de um derivado da sacarose espera-se que tenha um comportamento semelhante ao açúcar, por isso que nas formulações F10 e F19, seus resultados podem ser considerados semelhantes a formulação controle, sugerindo que em níveis menores de substituição não acarreta em prejuízo a estrutura dos bolos, o mesmo não pode ser dito das formulações de bolo contendo acessulfame K como substituto do açúcar, no qual os efeitos negativos da substituição foram observados logo nas formulações iniciais.

\section{0-CONCLUSÃO}

Conclui-se que o uso de ambos adoçantes acarretaram em prejuízos a estrutura dos bolos, pois alteraram negativamente o processo de gelatinização do amido nas massas de bolo prejudicando sua expansão e por fim o volume final do produto, onde o efeito foi mais pronunciado nos bolos contendo o acessulfame $\mathrm{K}$, indicando que entre os dois adoçantes utilizados ele é o menos indicado para tal finalidade.

\section{REFERENCIAS}

AACC International. Approved methods of the AACC. $10^{\text {th }}$ ed. AACC The Association: St Paul, Minnesotta, 2000.

ATTIA, E. A.; SHEHATA, H. A; ASKAR, A. An alternative formula for the sweetening of reduced-calorie cakes. Food Chem., v. 48, p.169-172, 1993.

BAEVA, M. R.; PANCHEV, I. N.; TERZIEVA, V. V. Comparative study of texture of normal and energy reduced sponge cakes. Narhung, v. 44, p. 242-246, 2000.

BAEVA, M. R.; TERZIEVA, V. V.; PANCHEV, I. N. Structural development of sucrosesweetened and sucrose-free sponge cakes during baking. Narhung, v.47, p. 154-160, 2003. 
BARNDT, R.L.; JACKSON G. Stability of sucralose in baked goods, Food Technol., V.44, p. 62-66, 1990.

BEMILLER, J.; WISTLER, R. Chemistry and thecnology. New York: Elservier, 2009.

BENNION, E.B.; BAMFORD, G. S. T. The Technology of Cake Making. London: Blackie Academic e Professional, 1997.

CAVALCANTE, R. S. Avaliação das características estruturais de bolos com redução calórica. Dissertação (Mestrado em Ciência e Tecnologia de Alimentos). Universidade Federal do Ceará, Fortaleza, 2012.

GAONKAR, A. G.; McPHERSON, A. Ingredient interactions: Effects on food quality. Taylor \&Francis, 2005.

LIN, S-D.; LEE, C-C. Qualities of chiffon cake prepared with indigestible dextrin and sucralose as replacement for sucrose. Cereal Chem., v. 82, p. 405-413, 2005.

KOCER, D.; HICSASMAZ, Z.; BAYINDIRLI, A.; KATNAS, S. Bubble and pore formation of the high-ratio cake formulation with polydextrose as a sugar- and fat-replacer. J. Food Eng., v. 78, p. 953-964, 2007.

MARTÍNEZ-CERVERA, S.; SANZ, T.; FISZMAN, SM. Rheological, textural and sensorial properties of low-sucrose muffins reformulated with sucralose/polydextrose. LWT - Food Sci. Technol., V. 45, n. 2, p. 213-220, 2012.

PSIMOULI, V.; OREOPOULOU, V. The effect of alternative sweeteners on batter rheology and cake properties. J. sci. Food Agric., v. 92, p. 99-105, 2012.

RONDA, F.; OLIETE, B. GOMEZ, CABALLERO, P. A., PANDO, V. Rheological study of layer cake batters made with soybean protein isolate and different starch sources. J. Food Eng., v. 102, p.272-277, 2011.

RONDA, F.; Manuel GÓMEZ, M.; Blanco, C. A.; CABALLERO, P. A. Effects of polyols and nondigestible oligosaccharides on the quality of sugar-free sponge cakes. Food Chem., v.90, p. 549455, 2005.

SCHIRMER, M.; JEKLEA, M.; ARENDTB, E.; BECKER, T. Physicochemical interactions of polydextrose for sucrose replacement in pound cake. Food Res. Int., V. 49, p. 291-298, 2012.

WILDERJANS, E.; PAREYT, B.;GOESAERT, H.; BRIJS, K.; DELCOUR, J. A. The role of gluten in a pound cake system: A model approach based on gluten-starch blends. Food Chem., v. 110, p. 909-915, 2008. 